\title{
BMJ Open Predicting opioid-induced oversedation in hospitalised patients: a multicentre observational study
}

\author{
John Garrett, ${ }^{1}$ Anneliese Vanston, ${ }^{2}$ Gerald Ogola, ${ }^{3}$ Briget da Graca (D) , ${ }^{3}$ \\ Cindy Cassity, ${ }^{2}$ Maria A Kouznetsova, ${ }^{4}$ Lauren R Hall, ${ }^{4}$ Taoran Qiu ${ }^{4}$
}

To cite: Garrett J, Vanston A, Ogola G, et al. Predicting opioid-induced oversedation in hospitalised patients: a multicentre observational study. BMJ Open

2021;11:e051663. doi:10.1136/ bmjopen-2021-051663

- Prepublication history for this paper is available online. To view these files, please visit the journal online (http://dx.doi org/10.1136/bmjopen-2021051663).

Received 24 March 2021 Accepted 27 October 2021

Check for updates

(c) Author(s) (or their employer(s)) 2021. Re-use permitted under CC BY-NC. No commercial re-use. See rights and permissions. Published by BMJ.

${ }^{1}$ Department of Emergency Medicine, Baylor University Medical Center, Dallas, Texas, USA

${ }^{2}$ Baylor University Medical Center, Dallas, Texas, USA

${ }^{3}$ Baylor Scott \& White Research Institute, Dallas, Texas, USA

${ }^{4}$ Baylor Scott \& White Health, Dallas, Texas, USA

Correspondence to

Dr John Garrett;

John.Garrett@bswhealth.org

\section{ABSTRACT}

Objectives Opioid-induced respiratory depression (OIRD) and oversedation are rare but potentially devastating adverse events in hospitalised patients. We investigated which features predict an individual patient's risk of OIRD or oversedation; and developed a risk stratification tool that can be used to aid point-of-care clinical decisionmaking.

Design Retrospective observational study.

Setting Twelve acute care hospitals in a large not-forprofit integrated delivery system.

Participants All inpatients $\geq 18$ years admitted between 1 July 2016 and 30 June 2018 who received an opioid during their stay (163190 unique hospitalisations).

Main outcome measures The primary outcome was occurrence of sedation or respiratory depression severe enough that emergent reversal with naloxone was required, as determined from medical record review; if naloxone reversal was unsuccessful or if there was no evidence of hypoxic encephalopathy or death due to oversedation, it was not considered an oversedation event. Results Age, sex, body mass index, chronic obstructive pulmonary disease, concurrent sedating medication, renal insufficiency, liver insufficiency, opioid naïvety, sleep apnoea and surgery were significantly associated with risk of oversedation. The strongest predictor was concurrent administration of another sedating medication (adjusted $\mathrm{HR}, 95 \% \mathrm{Cl}=3.88,2.48$ to 6.06 ); the most common such medications were benzodiazepines (29\%), antidepressants (22\%) and gamma-aminobutyric acid analogue (14.7\%). The c-statistic for the final model was 0.755 . The 24-point Oversedation Risk Criteria (ORC) score developed from the model stratifies patients as high ( $>20 \%, \geq 21$ points), moderate $(11 \%-20 \%, 10-20$ points) and low risk ( $\leq 10 \%$, $<10$ points)

Conclusions The ORC risk score identifies patients at high risk for OIRD or oversedation from routinely collected data, enabling targeted monitoring for early detection and intervention. It can also be applied to preventive strategies-for example, clinical decision support offered when concurrent prescriptions for opioids and other sedating medications are entered that shows how the chosen combination impacts the patient's risk.

\section{INTRODUCTION}

Opioid-induced respiratory depression (OIRD) and oversedation are rare but

\section{Strengths and limitations of this study}

This multi-hospital study is the first large study to develop a risk score for oversedation/opioid-induced respiratory depression that is applicable to all adult hospitalised patients prescribed opioids.

- All predictors used to build the novel 24-point Oversedation Risk Criteria score presented here are routinely collected and readily available from the electronic medical record; thus, implementation will not add to clinicians' data collection burden.

- The predictors include both patient characteristics that cannot be modified and treatment choices that can; it can therefore both facilitate targeted monitoring for early detection and intervention on oversedation/opioid-induced respiratory distress events, and be used in clinical decision support tools, providing information regarding the impact of concomitant medication choice on a patient's risk for such an event.

This is a novel risk score that should be validated in other, external case series.

frequently devastating side effects of opioid analgesia in hospitalised patients. In an analysis of closed malpractice claims, more than half the OIRD events resulted in death, and another $22 \%$ in severe brain damage. ${ }^{1}$ Furthermore, such events are highly preventable with improved monitoring and response. ${ }^{12}$ The challenge, however, lies in ensuring appropriate monitoring is provided. Opioid analgesia is the primary pharmacological intervention for managing pain in hospitalised patients, ${ }^{3}$ and more than half of all non-surgical patients admitted to hospitals, ${ }^{4}$ and almost all patients who undergo surgery, ${ }^{56}$ receive opioids during their stay. At these large volumes, continuous monitoring of all patients receiving opioids is not feasible: even if hospitals were to invest in the equipment necessary to provide pulse oximetry and capnography electronic monitoring ${ }^{7}$ for all patients receiving opioids, issues related to 
alarm fatigue and staff burden ${ }^{8}$ would remain significant barriers to effective monitoring.

Acknowledging the challenges to continuously monitoring all patients receiving opioids, clinical practice guidelines (eg, from the American Society of Anesthesiologists $^{9}$ and the American Society for Pain Management Nursing, ${ }^{10}$ ) as well as the Joint Commission accreditation requirements addressing safe use of opioids for pain management, ${ }^{11}$ include the step of identifying patients at high risk of OIRD or oversedation for enhanced monitoring. However, there is currently no agreed-upon method for assessing that risk. Multiple factors that increase patient risk-including patient demographic characteristics (such as older age ${ }^{12-14}$ and female sex, ${ }^{12} 1516$ ) clinical characteristics (such as cardiac disease, ${ }^{12} 1718$ pulmonary disease, ${ }^{12}{ }^{17}$ sleep apnoea, ${ }^{12}{ }^{15-20}$ diabetes, ${ }^{18}{ }^{20}$ impaired kidney function, ${ }^{12151618}$ and obesity ${ }^{121415}$ ) and opioidrelated factors (higher opioid dosage, ${ }^{12} 171920$ route of administration ${ }^{12} 16$ and concomitant use of other medications with sedative effects ${ }^{12} 13^{19}$ ). Survey data indicate that, while at least some of these factors are considered by most hospitals in identifying patients at high risk for OIRD and oversedation, there is substantial variation in which criteria are used. ${ }^{8}$ Furthermore, simply considering the list of possible risk factors does not help clinicians quantify actual risks for patients with multiple factors present, as it does not provide information regarding the extent to which they may be additive.

Some previous work has been done to synthesise multiple patient risk factors into clinically-useful risk scores. The PRODIGY trial (PRediction of Opioidinduced respiratory Depression In patients monitored by capnoGraphY), for example, developed a five-variable prediction model for OIRD, using data from a prospective trial in which participants were monitored continuously via capnography and pulse oximetry, but was limited to patients receiving parenteral opioids, treated on the general care floor and able to wear the continuous monitoring equipment. ${ }^{22} 23$ Another risk scoring system for severe opioid-related adverse events (including somnolence, respiratory depression and cardiopulmonary arrest) was developed from a US national cohort of medical hospitalisations, but did not consider surgical or trauma admissions. ${ }^{24} \mathrm{~A}$ risk index has also been developed and validated for serious OIRD or overdose among outpatients with opioid prescriptions, ${ }^{25}{ }^{26}$ but has not been tested for the inpatient setting (where dosages, routes of administration and the degree of control the patient has over when and how much of the medication to take, differ significantly) ${ }^{2526}$ What is thus currently missing from the literature is a risk score that is applicable to all hospitalised patients. Using data from our multi-hospital system, we sought to address this gap and (1) determine which features predict an individual patient's risk of OIRD or oversedation; and (2) develop a risk stratification tool to determine which patients are low, moderate and high risk for OIRD or oversedation that can be used at the point-ofcare to aid clinical decision-making.

\section{METHODS}

\section{Study population}

We considered all adult ( $\geq 18$ years) patients admitted to 1 of the 12 (healthcare system) acute care hospitals in North Texas between 1 July 2016 and 30 June 2018 who received an opioid during their inpatient stay.

\section{Outcomes}

The outcome of interest ('oversedation') was defined as an occurrence of sedation or respiratory depression severe enough that the primary care team felt emergent reversal with naloxone was indicated. Distinction was not made between three mechanisms through which opioid-induced ventilatory impairment (OIVI) occurs: depression of the respiratory centre in the brain stem, depression of the hypothalamus leading to increased arousal thresholds and sedation and decreased upper airway muscle tone leading to airway obstruction. Cases in which naloxone was administered were identified from the electronic medical record and individually reviewed by one of two healthcare providers via a standardised review process. Discrepancies between reviewers were discussed until both reviewers agreed with final determination. If naloxone administration successfully reversed the sedation event or opioid medication administered as part of inpatient care was determined to be the causative aetiology of sedation, the case was considered an oversedation event. Cases in which reversal with naloxone was not successful or in which the patient did not have evidence of hypoxic encephalopathy or death due to oversedation were not considered to be oversedation events. Patients who received naloxone during the course of procedural sedation were excluded from this analysis.

\section{Data collection}

All data were extracted from the electronic medical record. Data were collected on patient demographics, medical history and clinical and admission characteristics considered to be potential risk factors for oversedation, as identified in national guidelines. ${ }^{3}$ Patient outcomes (discharge disposition and length of stay) were also collected. All variables are listed in table 1 .

\section{Statistical analysis}

Patients' demographic characteristics, medical history and outcomes were summarised for the oversedated and not-oversedated groups. Continuous variables were summarised by mean and SD or median with IQR, while categorical variables were summarised by frequency and per cent. Differences in the characteristics were assessed by t-tests or Kruskal-Wallis tests for continuous data, and $\chi^{2}$ tests for categorical data.

Cox proportional hazards regression model was developed to predict in-hospital risk of oversedation. Survival times were defined as time from hospital admission to oversedation for patients that experienced oversedation, or time from hospital admission to discharge for patients that were never oversedated during their hospital stay. To 
Table 1 Patient demographic and clinical characteristics and outcomes for inpatients who did versus did not experience an opioid-related oversedation event

\begin{tabular}{|c|c|c|c|}
\hline & $\begin{array}{l}\text { Oversedation } \\
(\mathrm{N}=293)\end{array}$ & $\begin{array}{l}\text { No oversedation } \\
(\mathrm{N}=162897)\end{array}$ & $P$ value \\
\hline Age (years) & & & $<0.001$ \\
\hline$<50$ & $52(17.7 \%)$ & $63361(38.9 \%)$ & \\
\hline $50-59$ & $54(18.4 \%)$ & $26319(16.2 \%)$ & \\
\hline $60-69$ & $74(25.3 \%)$ & $31329(19.2 \%)$ & \\
\hline $70+$ & $113(38.6 \%)$ & $41888(25.7 \%)$ & \\
\hline Sex (female) & $191(65.2 \%)$ & $99562(61.1 \%)$ & 0.154 \\
\hline Race & & & 0.618 \\
\hline White & $230(78.5 \%)$ & $124351(76.3 \%)$ & \\
\hline Black & $46(15.7 \%)$ & $29139(17.9 \%)$ & \\
\hline Other & $17(5.8 \%)$ & $9407(5.8 \%)$ & \\
\hline Hispanic ethnicity & $34(11.6 \%)$ & $25330(15.5 \%)$ & 0.063 \\
\hline $\mathrm{BMI}-$ mean $\pm \mathrm{SD}\left(\mathrm{kg} / \mathrm{m}^{2}\right)$ & $30.1 \pm 10.3$ & $30.0 \pm 8.2$ & 0.745 \\
\hline Concurrent administration of sedating medication & $267(91.1 \%)$ & $95495(58.6 \%)$ & $<0.001$ \\
\hline Antihistamine & $73(24.9 \%)$ & $29952(18.4 \%)$ & 0.004 \\
\hline Renal insufficiency diagnosis & $141(48.1 \%)$ & $46415(28.5 \%)$ & $<0.001$ \\
\hline Liver insufficiency diagnosis & $189(64.5 \%)$ & $67917(41.7 \%)$ & $<0.001$ \\
\hline Chronic obstructive pulmonary disease & $73(24.9 \%)$ & $20592(12.6 \%)$ & $<0.001$ \\
\hline Heart failure & $72(24.6 \%)$ & $25506(15.7 \%)$ & $<0.001$ \\
\hline Thoracic or large incision that interferes with adequate ventilation & $20(6.8 \%)$ & $8577(5.3 \%)$ & 0.232 \\
\hline Positive sleep apnoea screen: Snore & $95(32.9 \%)$ & $40018(24.9 \%)$ & $<0.001$ \\
\hline Positive sleep apnoea screen: Doze off & $76(26.3 \%)$ & $22167(13.8 \%)$ & $<0.001$ \\
\hline Live alone & $4(1.4 \%)$ & $603(0.4 \%)$ & 0.018 \\
\hline Smoking history & & & 0.014 \\
\hline Non-smoker & $152(51.9 \%)$ & $95410(58.6 \%)$ & \\
\hline Former smoker & $90(30.7 \%)$ & $38785(23.8 \%)$ & \\
\hline Current smoker & $40(13.7 \%)$ & $24733(15.2 \%)$ & \\
\hline Unknown/missing & $11(3.8 \%)$ & $3969(2.4 \%)$ & \\
\hline Surgery & $148(50.5 \%)$ & $55262(33.9 \%)$ & $<0.001$ \\
\hline Untreated obstructive sleep apnoea & $55(19.4 \%)$ & $18846(12.0 \%)$ & $<0.001$ \\
\hline Opioid naïve & $155(52.9 \%)$ & $105519(64.8 \%)$ & $<0.001$ \\
\hline PCA basal & $7(2.4 \%)$ & $1066(0.7 \%)$ & $<0.001$ \\
\hline Days on opioids-median (Q1, Q3) & $7.0(4.0,13.0)$ & $3.0(2.0,5.0)$ & $<0.001$ \\
\hline Disposition & & & $<0.001$ \\
\hline Expired & $20(6.8 \%)$ & $4743(2.9 \%)$ & \\
\hline Home & $140(47.8 \%)$ & $128709(79.0 \%)$ & \\
\hline Transferred to other facilities & $133(45.4 \%)$ & $29445(18.1 \%)$ & \\
\hline Length of stay (days) - median (Q1, Q3) & $9.0(5.0,15.0)$ & $3.0(2.0,6.0)$ & $<0.001$ \\
\hline
\end{tabular}

BMI, body mass index; PCA, Patient Controlled Analgesia; Q, quartile.

develop the risk model, we first examined a full model with all potential risk factors of interest. Risk factors considered included age, sex, race, ethnicity, body mass index (BMI), smoking history, indicator of concurrent administration of sedating medication (defined as sedating medications administered either before or after administration of the opioid medication where the time that elapsed between administration was less than the predicted timeframe of the mechanism of action for the medication that was administered first), surgery, antihistamine, renal insufficiency, liver insufficiency, chronic obstructive pulmonary disease (COPD), heart failure 
$(\mathrm{HF})$, thoracic, sleep apnoea, live alone, untreated sleep apnoea, opioid naive and Patient Controlled Analgesia (PCA) basal rate. BMI was fitted with restricted cubic splines to account for any non-linear relationship with the outcome. ${ }^{27}$ The backward variable selection algorithm was implemented and risk factors significant at 0.25 level $(p<0.25)$ were initially retained. The final model was fitted using only previously retained variables and risk factors significant at 0.10 level $(p<0.10)$ were retained. The final prediction model was implemented in a nomogram to develop a risk score calculator for estimating probabilities of oversedation during hospitalisation for each individual. Predictive performance of the nomogram was validated for discrimination and calibration on the original data using 1000 bootstrap resamples. Discrimination was assessed by bootstrap-adjusted Harrell's Concordance Index (C-index) with 95\% CIs. ${ }^{28}$ Nomogram was developed using rms package of R V.3.5.0. Distribution of the relationship between probabilities of oversedation and risk scores were assessed and stratified as high, moderate and low risk.

Data analysis was conducted using SAS V.9.4 (SAS Institute) and R V.3.5.0 (The R Foundation for Statistical Computing) statistical programmes.

\section{Patient and public involvement}

There was no patient or public involvement in setting the research question or the outcome measures, in the design and implementation of the study or dissemination of results.

\section{RESULTS}

Between July 2016 and June 2018, a total of 163190 unique inpatient hospitalisations had documentation of opioid administration. Naloxone was found to have been

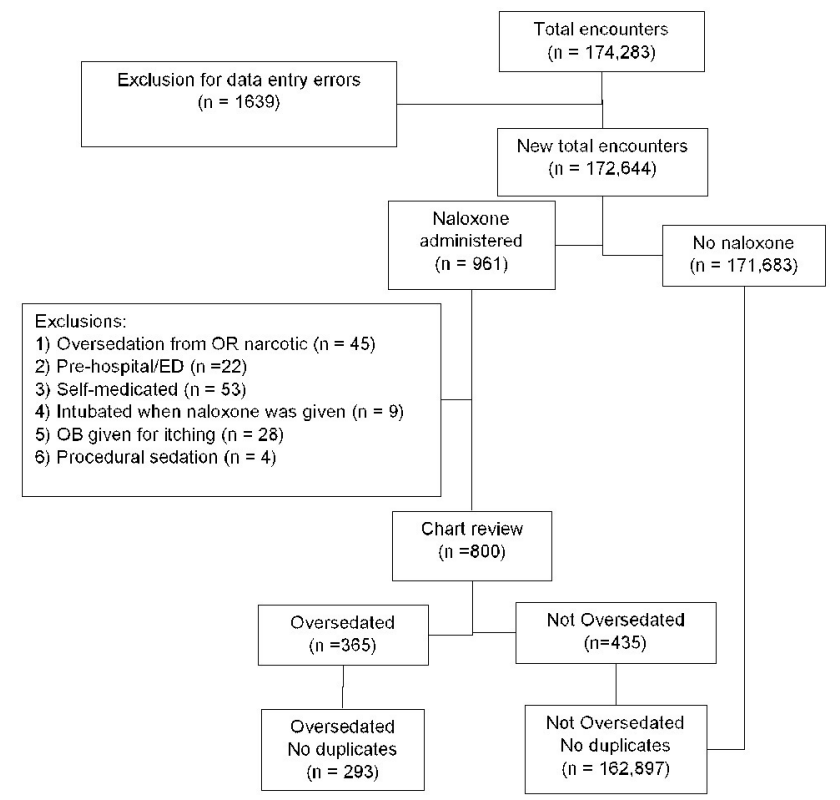

Figure 1 Inclusion and exclusion of hospital encounters for the development of the Oversedation Risk Criteria score. administered in 961 cases. As shown in figure 1, following exclusion of patients who received naloxone during procedural sedation, to treat a preadmission overdose, or for an indication other than oversedation, we identified $293(0.18 \%)$ hospitalisations with opioid administration that resulted in oversedation.

Table 1 compares patients characteristics between oversedated and non-oversedated groups. Bivariate analysis showed significant differences existed for most of the characteristics, except sex, race, ethnicity, average BMI and having a thoracic or large incision that interferes with adequate ventilation. In the multivariable model, age, sex, BMI, COPD, concurrent administration of sedating medication, renal insufficiency, liver insufficiency, opioid naïvety, sleep apnoea and surgery were significantly associated with risk of oversedation (table 2). The strongest predictor of oversedation was concurrent administration of another medication with sedative properties (adjusted HR, $95 \% \mathrm{CI}=3.88,2.48$ to 6.06 ); table 3 shows the medication types most frequently implicated. Older age was also significantly associated with increased risk of oversedation: patients' aged 60 years were more than 1.5 times higher likely to be oversedated when compared with those $<50$ years. BMI exhibited a non-linear relationship with the outcome, with low decreasing BMI $(<20)$ and high increasing BMI $(>35)$ both associated with significant increase in risk of oversedation (Figure 2). Opioid naïvety had a protective effect against oversedation.

Table 4 presents the points scores for risk factors in the final model. Point scores ranged from 0 to 10 while total risk score points ranged from 0 to 24. Figure 3 shows the risk of OIRD by Oversedation Risk Criteria (ORC) score. The predictive ability of the final model was very good with c-statistic $=0.755$.

\section{DISCUSSION}

In this study of 163191 hospitalised patients receiving opioids, we observed an incidence of oversedation of $0.18 \%$. The strongest predictor of oversedation was concurrent administration of other medications with sedative properties. Other strong predictors included older age, female sex, BMI, COPD, liver insufficiency, renal insufficiency, undergoing surgery and a history of sleep apnoea or positive sleep apnoea screen. Opioid naivety was protective against oversedation in our population. The predictive model developed showed good performance and was used to develop a points-based risk score, the ORC that can quickly inform clinicians regarding a patient's level of risk for oversedation.

Several of the predictors identified have good face validity for increasing risk for oversedation or respiratory distress. For example, COPD, in which the respiratory system is already compromised, and liver and renal insufficiency, in which clearance of some opioids or their metabolites may be reduced, increasing drug bioavailability to unsafe levels at dosages and frequencies that would be safe in the absence of dysfunction. ${ }^{29}{ }^{30}$ Likewise, 
Table 2 Associations between risk factors included in the full and reduced models for predicting opioid-induced respiratory depression or oversedation

\begin{tabular}{|c|c|c|}
\hline \multirow[b]{2}{*}{ Risk factors } & \multicolumn{2}{|l|}{ HR $(95 \% \mathrm{Cl})$ and $\mathrm{p}$ value } \\
\hline & Full model & Reduced model \\
\hline \multicolumn{3}{|l|}{ Age (years) } \\
\hline $50-59$ & 1.39 (0.91 to 2.13$), p=0.13$ & 1.39 (0.91 to 2.12$), p=0.13$ \\
\hline $60-69$ & 1.52 (1.01 to 2.28$), p=0.04$ & 1.54 (1.03 to 2.30$), p=0.03$ \\
\hline $70+$ & 1.69 ( 1.13 to 2.52$), p=0.01$ & 1.75 (1.19 to 2.57 ), $p=<0.01$ \\
\hline Sex (female vs male) & 1.59 (1.21 to 2.09$), p=<0.01$ & 1.60 (1.23 to 2.09$), p=<0.01$ \\
\hline \multicolumn{3}{|l|}{ Race } \\
\hline Black vs white & 0.83 (0.57 to 1.20$), p=0.31$ & \\
\hline Other vs white & $1.21(0.70$ to 2.10$), p=0.49$ & \\
\hline Hispanic ethnicity & 1.06 (0.71 to 1.58$), p=0.78$ & \\
\hline \multicolumn{3}{|l|}{ Smoking history } \\
\hline Former vs never & 1.03 (0.77 to 1.38$), p=0.84$ & \\
\hline Current vs never & 0.86 (0.58 to 1.28$), p=0.47$ & \\
\hline \multicolumn{3}{|l|}{$\mathrm{BMI}\left(\mathrm{kg} / \mathrm{m}^{2}\right)$} \\
\hline$(\mathrm{BMI}<26)$ & $1.02(1.00$ to 1.03$), p=0.07$ & 1.02 (1.00 to 1.04$), p=0.05$ \\
\hline$(\mathrm{BMI} \geq 26)$ & 0.68 (0.48 to 0.96$), p=0.03$ & 0.69 (0.49 to 0.97$), p=0.03$ \\
\hline Antihistamine & 0.91 (0.68 to 1.22$), p=0.53$ & \\
\hline Chronic obstructive pulmonary disease & 1.57 (1.14 to 2.15$), p=<0.01$ & $1.48(1.10$ to 1.99$), p=<0.01$ \\
\hline $\begin{array}{l}\text { Concurrent administration of sedating } \\
\text { medication }\end{array}$ & 3.89 (2.48 to 6.10$), p=<0.01$ & $3.88(2.48$ to 6.06$), p=<0.01$ \\
\hline Heart failure & 0.89 (0.64 to 1.22$), p=0.47$ & \\
\hline Live alone & 2.55 (0.63 to 10.29$), p=0.19$ & \\
\hline Liver insufficiency diagnosis & $1.60(1.21$ to 2.10$), p=<0.01$ & 1.62 (1.23 to 2.12$), p=<0.01$ \\
\hline Opioid naïve & 0.76 (0.59 to 0.97$), p=0.03$ & 0.74 (0.58 to 0.95$), p=0.02$ \\
\hline PCA basal & $1.87(0.82$ to 4.26$), p=0.13$ & 1.96 ( 0.87 to 4.46$), p=0.10$ \\
\hline Renal insufficiency & 1.40 (1.07 to 1.85$), p=0.02$ & 1.35 (1.03 to 1.76$), p=0.03$ \\
\hline Positive sleep apnoea screen (snore or doze-off) & 1.42 (1.08 to 1.87$), p=0.01$ & 1.45 (1.11 to 1.88$), p=<0.01$ \\
\hline Surgery & 1.57 ( 1.20 to 2.04$), p=<0.01$ & $1.53(1.18$ to 1.98$), p=<0.01$ \\
\hline $\begin{array}{l}\text { Thoracic or large incision that interferes with } \\
\text { adequate ventilation }\end{array}$ & 0.68 (0.41 to 1.12$), p=0.13$ & \\
\hline Untreated obstructive sleep apnoea & 1.18 (0.84 to 1.66$), p=0.34$ & \\
\hline
\end{tabular}

BMI, body mass index; PCA, Patient Controlled Analgesia.

concurrent use of other medications with sedating effects makes intuitive sense, as it complicates the balance of cumulative sedation against management of the pain, nausea or other symptoms each drug is prescribed to address. In the outpatient setting, coadministration of sedating agents has been shown to increase risk for overdose. ${ }^{31}$ Increasing age, presence of sleep apnoea and undergoing surgery, have all been previously identified as risk factors in the literature. ${ }^{17}$ In the case of age, physiological changes occur with ageing that affect how medicines are handled, including alterations in volumes of drug distribution, metabolism and clearance which can prolong half-life, increasing potential for drug toxicity and the likelihood of adverse drug reactions. ${ }^{32}$ Possible mechanisms that have been proposed for the impact of surgery include a combination of the residual effects of anaesthetic medications, as the risk appears to be greatest during the first 12-24 hours following surgery. ${ }^{17}$ With respect to sleep apnoea, it is a prevalent characteristic among patients who die due to critical respiratory events during the first 24 hours following surgery, ${ }^{18}$ and among patients who suffer postoperative OIRD. ${ }^{1}$ The intermittent hypoxia that is a component of obstructive sleep apnoea has been shown to both increase pain and enhance opioid effects; in addition, opioids attenuate the arousal response to hypoxia and prolong airway obstruction, a combination of effects that has been hypothesised as explaining the association between sleep apnoea and 
Table 3 Medications with sedating properties prescribed concomitantly with opioids in patients who experienced an opioid-related oversedation event

\begin{tabular}{lr}
\hline Drug type/category & n (\%) \\
\hline Benzodiazepine & $245(29.0)$ \\
\hline Antidepressants & $186(22.0)$ \\
Gamma-aminobutyric acid analogue & $124(14.7)$ \\
$\begin{array}{l}\text { Miscellaneous anxiolytic, sedative and } \\
\text { hypnotic (sleep aids) }\end{array}$ & $86(10.2)$ \\
Antipsychotic & $69(8.2)$ \\
Antihistamine & $68(8.0)$ \\
Anticonvulsant & $38(4.5)$ \\
Dopaminergic anti-Parkinsonism agents & $15(1.8)$ \\
Barbiturate & $6(0.7)$ \\
Phenothiazine antiemetics & $5(0.6)$ \\
Carbonic anhydrase inhibitor & $3(0.4)$ \\
\hline
\end{tabular}

risk for OIRD. ${ }^{1733}$ Our findings regarding the risk associated with BMI are unique in that, while previous studies have found increased risk of oversedation or OIRD with obesity ${ }^{12}$ ours is the first to elucidate the J-shaped curve in which risk increases as BMI values move away in either direction from the point of inversion at $26 \mathrm{~kg} / \mathrm{m}^{2}-\mathrm{al}-$ though a similar relationship between BMI and risk for adverse outcomes has been shown in other contexts. ${ }^{27}$

The low incidence of oversedation observed here was in line with previous reports examining similarly severe opioid-related adverse events (ie, necessitating the administration of a reversal agent such as naloxone). The frequency of such events varies according to the population studied-for example, reported at $0.4 \%$ in hospitalised medical patients receiving opioids, ${ }^{24} 0.1 \%$ for postoperative patients, ${ }^{34} 0.3 \%$ for patients undergoing major surgery ${ }^{35}$ and $\leq 0.07 \%$ in women who had

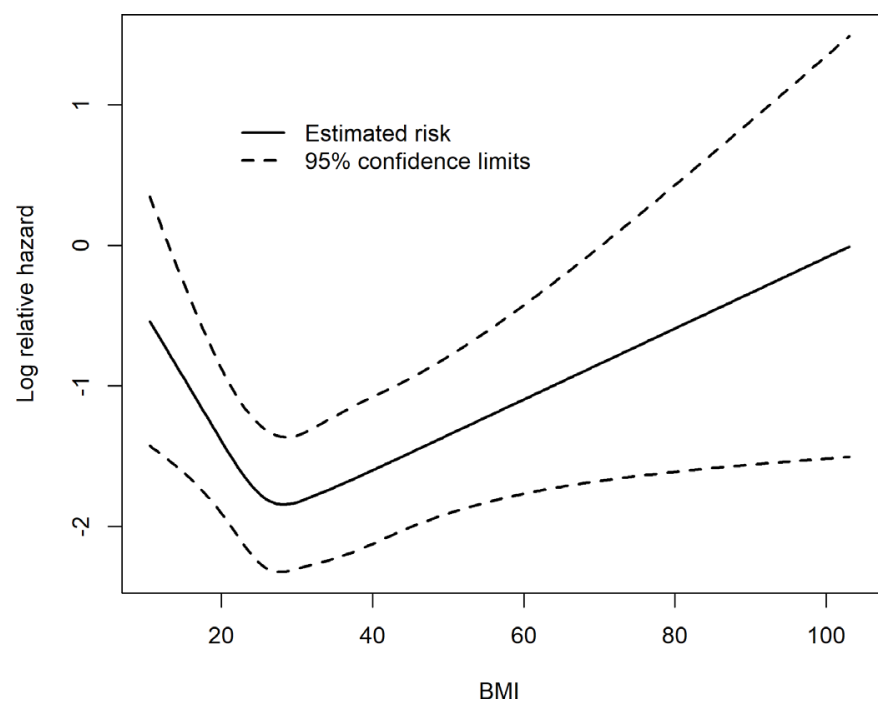

Figure 2 Risk of opioid-induced respiratory depression or oversedation by body mass index (BMI).
Table 4 Points assigned per risk factor in the Oversedation Risk Criteria score

\begin{tabular}{|c|c|}
\hline Risk factor & Points \\
\hline \multicolumn{2}{|l|}{ Age (years) } \\
\hline$<50$ & 0 \\
\hline $50-59$ & 1 \\
\hline $60+$ & 2 \\
\hline Sex-female & 2 \\
\hline \multicolumn{2}{|l|}{ Body mass index $\left(\mathrm{kg} / \mathrm{m}^{2}\right)$} \\
\hline 10 & 5 \\
\hline 20 & 2 \\
\hline 30 & 0 \\
\hline 40 & 1 \\
\hline 50 & 2 \\
\hline 60 & 3 \\
\hline 70 & 4 \\
\hline 80 & 5 \\
\hline 90 & 6 \\
\hline 100 & 7 \\
\hline 110 & 8 \\
\hline 120 & 9 \\
\hline 130 & 10 \\
\hline Concurrent administration of sedating medication & 5 \\
\hline Renal insufficiency & 1 \\
\hline Liver insufficiency & 2 \\
\hline Chronic obstructive pulmonary disease & 1 \\
\hline Sleep apnoea & 2 \\
\hline Surgery within 24 hours & 2 \\
\hline Not opioid naïve & 1 \\
\hline PCA basal & 3 \\
\hline
\end{tabular}

PCA, Patient Controlled Analgesia.

undergone caesarean. ${ }^{36}$ Previous studies have also set out to develop risk prediction models for OIRD or oversedation. These include a small case-control study from a US community hospital considering all hospitalised patients receiving opioids, ${ }^{16}$ a large observational study of medical patients hospitalised in the USA receiving opioids ${ }^{24}$ and a risk score developed from an international prospective trial (PRODIGY) of the use of continuous capnography and oximetry to monitor patients receiving opioids on general inpatient wards. ${ }^{22}$ One additional case-control study by Pawasauskas $e t a l^{37}$ did not develop a risk model per se, but identified a set of risk factors and found that patients with a higher number of these factors were more likely to experience oversedation. Predictors included in these previous models both overlapped with and differed from the predictors used in our risk score. Older age was consistently identified as a risk factor, and some measure related to sleep apnoea was included as a risk 


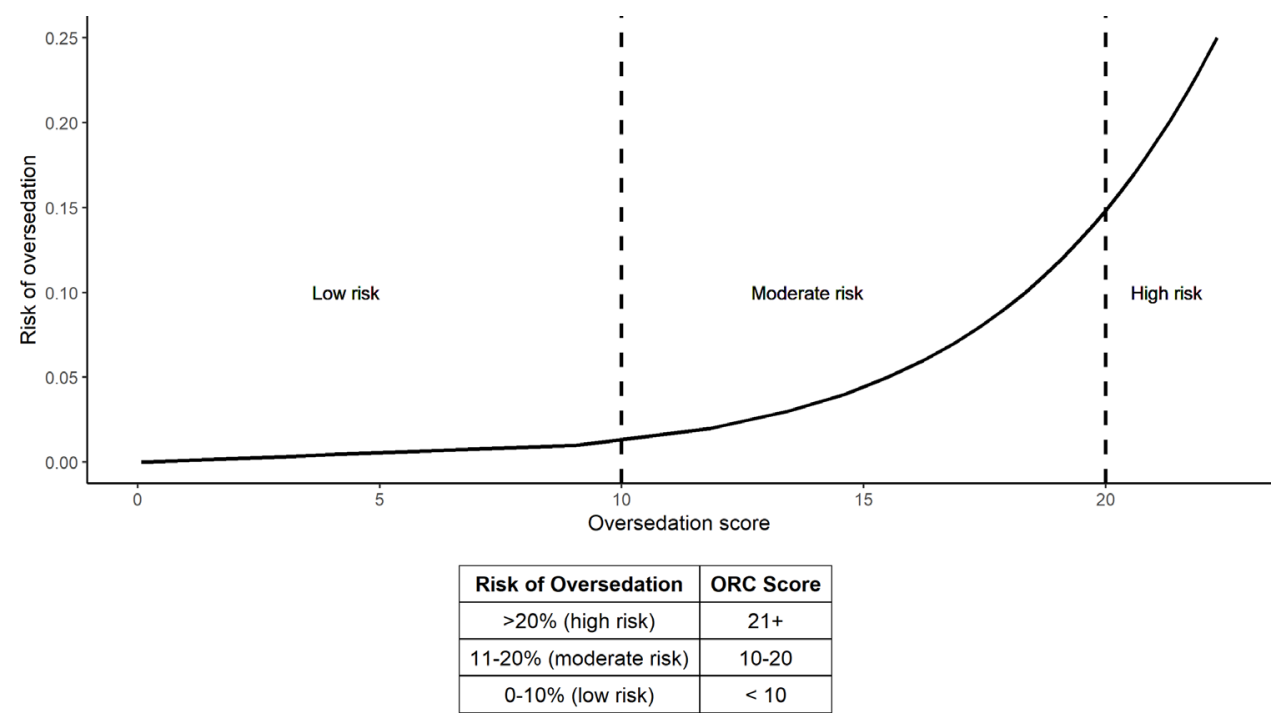

Figure 3 Risk of opioid-induced respiratory depression or oversedation by Oversedation Risk Criteria (ORC) score.

factor (sleep disorders, ${ }^{22}$ untreated sleep apnoea ${ }^{16}$ or obstructive sleep apnoea ${ }^{24}$ ) in all but Pawasauskas et al. ${ }^{37}$ Two of the previous risk models, as well as Pawasauskas et $a l,{ }^{37}$ included factors related to the renal insufficiency variable in our risk model (comorbid renal disease ${ }^{16}$ and renal failure on admission ${ }^{24}$ ), but only Pawasauskas et $a l^{37}$ (hepatic disease) and the risk model developed for medical hospitalisations (hepatic failure on admission $^{24}$ ) included any measure similar to the liver insufficiency variable in our model. These were also the only two models to include measures related to our strongest predictor of oversedation: concurrent administration of other medications with sedative properties (concurrent sedating medications, ${ }^{37}$ and short-acting benzodiazepine exposure and antipsychotic exposure ${ }^{24}$ ). While sex was included in all models except Pawasauskas $e t ~ a l,{ }^{37}$ two of the previous studies identified female sex as associated with increased risk ${ }^{1624}$ (as in our model) while the third identified male sex as being so associated. ${ }^{22}$ Similarly, opioid naïvety was included in the PRODIGY risk score as a risk factor, ${ }^{22}$ while in our model, and in Pawasauskas et $a l,{ }^{37}$ it was a protective characteristic. This discordance between studies requires further investigation, including into the relative opioid doses received by naive versus non-naive patients, and the default dosing applied in the clinical setting being studied. It may be that opioid naïvety is protective in the real-world practice settings where clinicians know to use a lower default starting dosage to effectively control pain in patients who have not developed tolerance. In contrast, non-opiate naive patients may have experienced increased risk of OIVI from higher starting doses and increased rate of escalating supplemental doses needed to control pain. Of the remaining predictors in our model, Pawasauskas $e t a l^{37}$ was the only previous model to include BMI and respiratory disease. None of the previous models included surgery (understandable for the risk model targeting medical hospitalisations only ${ }^{24}$ ), or PCA basal rate (although one did identify receipt of long-acting oxycodone or as-needed hydromorphone as a risk factor, which is physiologically similar). ${ }^{16}$ Risk factors they identified and included that were not a part of our model include congestive $\mathrm{HF}^{22}{ }^{24}$ psychosis or depression, ${ }^{24}$ opioid abuse/dependence, ${ }^{24}$ non-opioid drug abuse/dependence ${ }^{24}$ and presence on admission of respiratory failure, shock/hypotension on admission, acidosis or neurological failure. ${ }^{24}$ Despite these differences, the performance of our risk model and the previous models is similar: both the PRODIGY model reported and the risk model targeting medical hospitalised patients reported c-statistics of $0.68-0.71,{ }^{22} 24$ which our model modestly outperformed at 0.755 , while the remaining risk model, which was developed from a small single centre study, achieved a c-statistic of $0.86 .^{16}$ The PRODIGY score showed greater separation in risk for OIRD between its low, medium and high risk score categories $\left(24 \%, 42 \%\right.$ and $65 \%^{22}$ compared with our $<5 \%, 5 \%-15 \%$ and $>15 \%)$, but this is likely explained by their having examined respiratory depression detected by continuous pulse oximetry and capnography monitoring, rather than the clinically relevant potentially life-threatening events requiring naloxone reversal we examined.

Some limitations should be kept in mind when interpreting our results. First, while our study sample was large and drawn from 12 acute care hospitals, it was nonetheless drawn from a single healthcare system. To the extent that risk for oversedation is affected by institutional prescribing policies or clinical decision support tools, monitoring schedules/equipment/staffing resources and similar structural considerations governed at the healthcare system level, our findings may be less generalisable to all other settings. The differences noted in the risk factors included among the risk scores that have been developed to date-with all models showing good discrimination-indicates that there is further work to be done explain these discrepancies. Second, as was noted by 
the developers of a previous risk score focusing on oversedation or OIRD events identified through naloxone reversal, ${ }^{24}$ the rarity of this adverse outcome keeps the positive predictive value of even a risk score with good discrimination relatively low. Thus, while the risk score offers important value in terms of identifying patients at increased risk for OIRD or oversedation, care must still be taken in selecting mechanisms for mitigating that risk, to avoid issues such as alarm fatigue that have been reported as barriers to increased monitoring (human or electronic) to catch earlier signs of oversedation and prevent its progression. ${ }^{8}$ Another factor to be kept in mind with identifying OIRD events through naloxone reversal in an retrospective study is that staff may have had inherent biases related to their perceptions of patients' risk that made them more likely to diagnose oversedation and administer naloxone in some groups than others that cannot be captured in our data. However, such effects are likely to be small, and do reflect the real-word setting in which this risk score was developed and will be applied.

Recommendations included in national guidelines on monitoring for OIRD and oversedation include that 'all patients who will receive opioids undergo a comprehensive assessment of level of individual risk before initiation of opioid therapy'. ${ }^{10}$ While the guidelines go on to suggest that a risk factor checklist be integrated into the electronic health record to assist with this comprehensive assessment and its documentation, no guidance is provided on how clinicians should judge cumulative risk for patients in whom multiple risk factors are present. The ORC risk score we have developed is a tool to achieve this, and unlike previous studies, is applicable to all adult patients admitted to an acute care hospital. Future research should include evaluation of the ORC risk score for use in ambulatory centres: as more procedures that have traditionally been performed in the high resource setting of acute care hospitals transition to such settings, ${ }^{38}$ where the availability of staff and resources to monitor patients for or respond to events of OIRD or oversedation may be more limited, identification of high-risk patients may be even more critical for maintaining patient safety.

Beyond application to targeted monitoring of high-risk patients for early detection of and intervention to prevent progression of OIRD and oversedation events, our results suggest opportunities for prevention through interventions aimed at prescribing clinicians-for example, clinical decision support using the ORC offered when concurrent prescriptions for opioids and other sedating medications are entered. Such preventive approaches will be critical to achieving sustained improvement, as the high rate of false positive alarms-even among patients at high risk for OIRD and oversedation-leave strategies that focus exclusively on monitoring and early detection vulnerable to alarm fatigue and competing priorities for attention among staff tasked with responding.

Contributors JG (study guarantor): study concept, study design, data analysis, data interpretation, drafting the manuscript. AV: data collection, data management, revising the manuscript. BdG: literature search, drafting the manuscript. G0: data collection, data analysis, figures and tables, drafting the manuscript. MAK: study concept, revising the manuscript. CC: data collection, revising the manuscript. LRH: data collection, data management, revising the manuscript. TQ: data collection, data management, revising the manuscript.

Funding This work was funded by the Baylor Health Care System Foundation. The funder played no role in this study, and the research team operated independently.

Competing interests None declared.

Patient and public involvement Patients and/or the public were not involved in the design, or conduct, or reporting, or dissemination plans of this research.

Patient consent for publication Not applicable.

Ethics approval This study involves human participants and was approved by the Baylor Scott \& White Research Institute Institutional Review Board (IRB 018-761) with a waiver of informed consent.

Provenance and peer review Not commissioned; externally peer reviewed.

Data availability statement Data are available upon reasonable request. Data sharing: Please contact the corresponding author with any requests.

Open access This is an open access article distributed in accordance with the Creative Commons Attribution Non Commercial (CC BY-NC 4.0) license, which permits others to distribute, remix, adapt, build upon this work non-commercially, and license their derivative works on different terms, provided the original work is properly cited, appropriate credit is given, any changes made indicated, and the use is non-commercial. See: http://creativecommons.org/licenses/by-nc/4.0/.

ORCID iD

Briget da Graca http://orcid.org/0000-0002-8634-7122

\section{REFERENCES}

1 Lee LA, Caplan RA, Stephens LS, et al. Postoperative opioid-induced respiratory depression: a closed claims analysis. Anesthesiology 2015;122:659-65.

2 The Joint Commission. Safe use of opioids in hospitals. Sentinel Event Alert 2012;49:1-5.

3 Jarzyna D, Jungquist CR, Pasero C, et al. American Society for pain management nursing guidelines on monitoring for opioidinduced sedation and respiratory depression. Pain Manag Nurs 2011;12:e10:118-45.

4 Herzig SJ, Rothberg MB, Cheung M, et al. Opioid utilization and opioid-related adverse events in nonsurgical patients in US hospitals. J Hosp Med 2014;9:73-81.

5 Kessler ER, Shah M, Gruschkus SK, et al. Cost and quality implications of opioid-based postsurgical pain control using administrative claims data from a large health system: opioid-related adverse events and their impact on clinical and economic outcomes. Pharmacotherapy 2013;33:383-91.

6 Minkowitz HS, Gruschkus SK, Shah M, et al. Adverse drug events among patients receiving postsurgical opioids in a large health system: risk factors and outcomes. Am J Health Syst Pharm 2014:71:1556-65.

7 Gupta RA, Edwards DA. Monitoring for opioid-induced respiratory depression. APSF News/ 2018;32:70-2 https://www.apsf.org/article/ monitoring-for-opioid-induced-respiratory-depression/

8 Jungquist CR, Willens JS, Dunwoody DR, et al. Monitoring for opioid-induced advancing sedation and respiratory depression: ASPMN membership survey of current practice. Pain Manag Nurs 2014;15:682-93.

9 Practice guidelines for the prevention, detection, and management of respiratory depression associated with neuraxial opioid administration: an updated report by the American Society of Anesthesiologists Task force on neuraxial opioids and the American Society of regional anesthesia and pain medicine. Anesthesiology 2016;124:535-52.

10 Jungquist CR, Quinlan-Colwell A, Vallerand A, et al. American Society for pain management nursing guidelines on monitoring for opioid-induced advancing sedation and respiratory depression: revisions. Pain Manag Nurs 2020;21:7-25.

11 Joint Commission enhances pain assessment and management requirements for accredited hospitalsVol 37201724

12 Gupta K, Prasad A, Nagappa M, et al. Risk factors for opioid-induced respiratory depression and failure to rescue: a review. Curr Opin Anaesthesiol 2018;31:110-9. 
13 Gordon DB, Pellino TA. Incidence and characteristics of naloxone use in postoperative pain management: a critical examination of naloxone use as a potential quality measure. Pain Manag Nurs 2005:6:30-6.

14 Overdyk FJ, Carter R, Maddox RR, et al. Continuous oximetry/ capnometry monitoring reveals frequent desaturation and bradypnea during patient-controlled analgesia. Anesth Analg 2007;105:412-8.

15 Overdyk F, Dahan A, Roozekrans M, et al. Opioid-induced respiratory depression in the acute care setting: a compendium of case reports. Pain Manag 2014;4:317-25.

16 Brant JM, Stringer L, Jurkovich LR, et al. Predictors of oversedation in hospitalized patients. Am J Health Syst Pharm 2018;75:1378-85.

17 Gupta K, Nagappa M, Prasad A, et al. Risk factors for opioid-induced respiratory depression in surgical patients: a systematic review and meta-analyses. BMJ Open 2018;8:e024086.

18 Ramachandran SK, Haider N, Saran KA, et al. Life-threatening critical respiratory events: a retrospective study of postoperative patients found unresponsive during analgesic therapy. J Clin Anesth 2011;23:207-13.

19 Weingarten TN, Herasevich V, McGlinch MC, et al. Predictors of delayed postoperative respiratory depression assessed from naloxone administration. Anesth Analg 2015;121:422-9.

20 Weingarten TN, Chong EY, Schroeder DR, et al. Predictors and outcomes following naloxone administration during phase I anesthesia recovery. J Anesth 2016;30:116-22.

21 Overdyk FJ, Dowling O, Marino J, et al. Association of opioids and sedatives with increased risk of in-hospital cardiopulmonary arrest from an administrative database. PLoS One 2016;11:e0150214.

22 Khanna AK, Bergese SD, Jungquist CR, et al. Prediction of opioid-induced respiratory depression on inpatient wards using continuous capnography and oximetry: an international prospective, observational trial. Anesth Analg 2020;131:1012-24.

23 Khanna AK, Overdyk FJ, Greening C, et al. Respiratory depression in low acuity Hospital settings-seeking answers from the prodigy trial. $J$ Crit Care 2018;47:80-7.

24 Herzig SJ, Stefan MS, Pekow PS, et al. Risk factors for severe opioid-related adverse events in a national cohort of medical hospitalizations. J Gen Intern Med 2020;35:538-45.

25 Zedler B, Xie L, Wang L, et al. Development of a risk index for serious prescription opioid-induced respiratory depression or overdose in veterans' health administration patients. Pain Med 2015;16:1566-79.
26 Zedler BK, Saunders WB, Joyce AR, et al. Validation of a screening risk index for serious prescription opioid-induced respiratory depression or overdose in a US commercial health plan claims database. Pain Med 2018;19:68-78.

27 Filardo G, Hamilton C, Hamman B, et al. Categorizing BMI may lead to biased results in studies investigating in-hospital mortality after isolated CABG. J Clin Epidemiol 2007;60:1132-9.

28 Harrell FE. Regression modeling strategies: with applications to linear models, logistic regression and survival analysis. New York: SpringerVerlag, 2001.

29 Bosilkovska M, Walder B, Besson M, et al. Analgesics in patients with hepatic impairment: pharmacology and clinical implications. Drugs 2012;72:1645-69.

30 Dean M. Opioids in renal failure and dialysis patients. $J$ Pain Symptom Manage 2004;28:497-504.

31 Sun EC, Dixit A, Humphreys K, et al. Association between concurrent use of prescription opioids and benzodiazepines and overdose: retrospective analysis. BMJ 2017;356:j760.

32 Davies EA, O'Mahony MS. Adverse drug reactions in special populations - the elderly. Br J Clin Pharmacol 2015;80:796-807.

33 Lam KK, Kunder S, Wong J, et al. Obstructive sleep apnea, pain, and opioids: is the riddle solved? Curr Opin Anaesthesiol 2016;29:134-40.

34 Khelemsky Y, Kothari R, Campbell N, et al. Incidence and demographics of post-operative naloxone administration: a 13-year experience at a major tertiary teaching institution. Pain Physician 2015;18:E827-9.

35 Cashman JN, Dolin SJ. Respiratory and haemodynamic effects of acute postoperative pain management: evidence from published data. Br J Anaesth 2004;93:212-23.

36 Crowgey TR, Dominguez JE, Peterson-Layne $\mathrm{C}$, et al. A retrospective assessment of the incidence of respiratory depression after neuraxial morphine administration for postcesarean delivery analgesia. Anesth Analg 2013;117:1368-70.

37 Pawasauskas J, Stevens B, Youssef R, et al. Predictors of naloxone use for respiratory depression and oversedation in hospitalized adults. Am J Health Syst Pharm 2014;71:746-50.

38 DeCook CA. Outpatient joint arthroplasty: transitioning to the ambulatory surgery center. J Arthroplasty 2019;34:S48-50. 